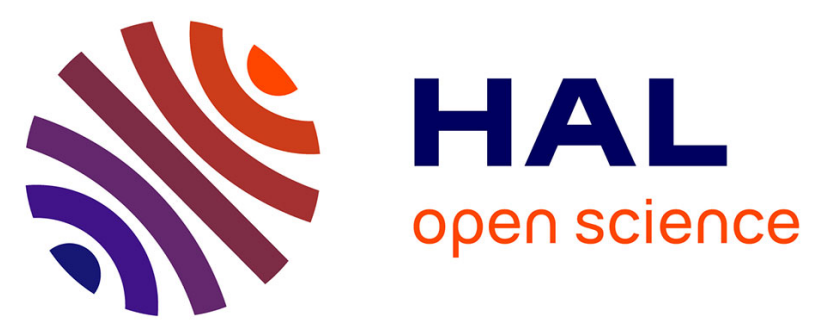

\title{
Characterization of new monoclonal PF4-specific antibodies as useful tools for studies on typical and autoimmune heparin-induced thrombocytopenia
}

Caroline Vayne, Thi-Huong Nguyen, Jérôme Rollin, Noémie Charuel, Anne

Poupon, Claire Pouplard, Nicole Normann, Yves Gruel, Andreas Greinacher

\section{To cite this version:}

Caroline Vayne, Thi-Huong Nguyen, Jérôme Rollin, Noémie Charuel, Anne Poupon, et al.. Characterization of new monoclonal PF4-specific antibodies as useful tools for studies on typical and autoimmune heparin-induced thrombocytopenia. Thrombosis and Haemostasis, 2021, 121 (3), pp.322-331. 10.1055/s-0040-1717078 . hal-03039816

\section{HAL Id: hal-03039816 \\ https://cnrs.hal.science/hal-03039816}

Submitted on 9 Dec 2020

HAL is a multi-disciplinary open access archive for the deposit and dissemination of scientific research documents, whether they are published or not. The documents may come from teaching and research institutions in France or abroad, or from public or private research centers.
L'archive ouverte pluridisciplinaire HAL, est destinée au dépôt et à la diffusion de documents scientifiques de niveau recherche, publiés ou non, émanant des établissements d'enseignement et de recherche français ou étrangers, des laboratoires publics ou privés. 


\title{
Characterization of New Monoclonal PF4-Specific Antibodies as Useful Tools for Studies on Typical and Autoimmune Heparin-Induced Thrombocytopenia
}

\author{
Caroline Vayne ${ }^{1,2, *}$ Thi-Huong Nguyen ${ }^{3,4, *}$ Jérôme Rollin ${ }^{1,2}$ Noémie Charuel ${ }^{1}$ Anne Poupon ${ }^{5,6}$ \\ Claire Pouplard ${ }^{1,2}$ Nicole Normann ${ }^{3}$ Yves Gruel ${ }^{1,2, * *} \quad$ Andreas Greinacher ${ }^{3, * *}$
}

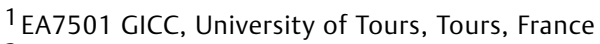

${ }^{2}$ Department of Haemostasis, Regional University Hospital Centre Tours, Tours, France

3 Institute for Immunology and Transfusion Medicine, University Medicine Greifswald, Greifswald, Germany

${ }^{4}$ Institute for Bioprocessing and Analytical Measurement Techniques, Heilbad Heiligenstadt, Germany

5 PRC, INRA, CNRS, University of Tours, Nouzilly, France

${ }^{6}$ MAbSilico SAS, Nouzilly, France

Thromb Haemost

\author{
Address for correspondence Andreas Greinacher, MD, Institut für \\ Immunologie und Transfusionsmedizin, Universitätsmedizin \\ Greifswald, Sauerbruchstrasse, 17475 Greifswald, Germany \\ (e-mail: Andreas.Greinacher@med.uni-greifswald.de). \\ Yves Gruel, MD-PhD, Department of Haemostasis, Regional University \\ Hospital of Tours, Chambray-Lès-Tours Indre-et-Loire 37170, Tours, \\ France (e-mail: yves.gruel@univ-tours.fr).
}

\section{Abstract}

\section{Keywords}

- platelet factor 4

- monoclonal antibodies

- heparin

- thrombocytopenia
Background Heparin-induced thrombocytopenia (HIT) is typically caused by plateletactivating immunoglobulin $\mathrm{G}(\mathrm{IgG})$ antibodies (Abs) against platelet factor 4 (PF4) complexed with heparin $(\mathrm{H})$. Much less frequent "autoimmune" HIT is distinguished from typical HIT by platelet activation without heparin and the presence of both antiPF4/H and anti-PF4 IgG. We developed three murine monoclonal anti-PF4 Abs with a human Fc-part, 1E12, 1C12, and 2E1, resembling autoimmune HIT Abs.

Objectives To characterize $1 \mathrm{E} 12,1 \mathrm{C} 12$, and $2 \mathrm{E} 1$ in comparison to the heparindependent monoclonal anti-PF4/H Abs 5B9 and KKO, and polyclonal Abs from patients with typical HIT (group-2) and autoimmune HIT (group-3).

Methods Interactions of Abs with PF4 and PF4/H were studied by enzyme-linkedimmunosorbent assay, single-molecule force spectroscopy, isothermal titration calorimetry, and dynamic light scattering. Serotonin release assay and heparin-induced platelet activation assay were used to assess platelet activation. The binding sites of monoclonal Abs on PF4 were predicted in silico (MAbTope method).

Results $1 \mathrm{C} 12,1 \mathrm{E} 12$, and $2 \mathrm{E} 1$ displayed higher affinity for PF4/H complexes than $5 \mathrm{~B} 9$ and KKO, comparable to human group-3 Abs. Only 1C12, 1E12, 2E1, and group-3 Abs formed large complexes with native PF4, and activated platelets without heparin. The predicted binding sites of $1 \mathrm{C} 12,1 \mathrm{E} 12$, and $2 \mathrm{E} 1$ on PF4 differed from those of KKO and $5 \mathrm{~B} 9$, but were close to each other. $2 \mathrm{E} 1$ exhibited unique bivalent binding, involving its antigen recognition site to PF4 and charge-dependent interactions with heparin.

Conclusion 1C12, 1E12, and 2E1 are tools for studying the pathophysiology of autoimmune HIT. 2E1 provides evidence for a new binding mechanism of HIT Abs.

C.V and T.H.N. contributed equally to the study.

received

March 9, 2020

accepted after revision

August 13, 2020 (c) Georg Thieme Verlag KG

Stuttgart · New York
DOI https://doi.org/

$10.1055 / \mathrm{s}-0040-1717078$.

ISSN 0340-6245. 


\section{Introduction}

Heparin-induced thrombocytopenia (HIT) is an adverse drug effect caused in most patients by platelet-activating immunoglobulin $\mathrm{G}$ (IgG) antibodies (Abs) directed against platelet factor 4 (PF4-specific Abs). ${ }^{1}$ Binding of PF4 to heparin (H) induces its conformational change ${ }^{2}$ and exposes the binding site(s) for heparin-dependent PF4-specific Abs (anti-PF4/H Abs). In addition to Abs with PF4/H specificity, patients can develop Abs, which bind PF4 and activate platelets in the absence of heparin (anti-PF4 Abs). This results in a spectrum of symptoms in patients with PF4-specific Abs, ranging from asymptomatic serum positivity to life-threatening autoimmune HIT. ${ }^{3,4}$

Major progress has been made in understanding the pathogenesis of HIT in recent years. ${ }^{2,5,6}$ However, mechanistic studies with human Abs obtained from patients have limitations as these sera always contain a polyclonal mixture of Abs with different binding characteristics. ${ }^{7}$ Purification of $\mathrm{Ab}$ subgroups from these sera is possible but requires major efforts. ${ }^{6}$ Therefore, the availability of well-characterized monoclonal Abs (moAbs) will likely allow development of a wide variety of new experimental approaches.

To date, only one murine monoclonal anti-PF4/H Ab, KKO that mimics the biological function of human HIT Abs, is commercially available. KKO activates platelets and monocytes by cross-linking FcyRIla, but it is an IgG2b Ab with an Fc-fragment, which differs from those of human $\operatorname{IgG}(\mathrm{hIgG})^{8}{ }^{8}$ Also, the binding characteristics of the $F\left(a b^{\prime}\right)_{2}$-part of KKO differ from the ones evidenced with human HIT $\operatorname{IgG} .{ }^{9}$ More recently, we developed 5B9, a moAb to PF4/H complexes with a human Fc fragment, and which fully mimics the cellular effects of human heparin-dependent HIT Abs. ${ }^{10}$

Some murine monoclonal anti-PF4 Abs are commercially available, but only very few are well characterized. In addition, none of them possess a human Fc fragment. In this context, we have developed, besides 5B9 (an anti-PF4/H Ab), three anti-PF4 murine moAbs with a human Fc fragment, $1 \mathrm{E} 12,1 \mathrm{C} 12$, and 2E1. These new moAbs exhibit characteristics very similar to those previously defined for human autoimmune HIT Abs, ${ }^{6}$ and could be useful tools for studying the pathophysiology of atypical forms of HIT.

\section{Methods}

\section{Production of Monoclonal PF4-Specific Antibodies}

The four moAbs (5B9, 1E12, 1C12, and 2E1) have been obtained as previously described. ${ }^{10}$ Briefly, transgenic Balb/c mice homozygous for the $\mathrm{C}$ gene of human $\operatorname{IgG}$ that directly produce chimeric IgG1 Abs (GammaPrim, B Cell Design, Limoges, France) were injected intraperitoneally with preformed PF4/ heparin complexes (human PF4; $50 \mu \mathrm{g}$, Hyphen BioMed) and unfractionated heparin (UFH $2 \mathrm{IU} / \mathrm{mL}$; Heparin Choay, Sanofi). Mice were sacrificed and their spleens were removed for fusion procedures, hybridomas were cultured for 13 days, and supernatants were screened by a homemade enzymelinked-immunosorbent assay (ELISA), adapted from Arepally et $\mathrm{al}^{8}{ }^{8}$ using microplates coated with purified human PF4 (2.5 $\mu \mathrm{g} / \mathrm{mL}$, Hyphen Biomed, Neuville-sur-Oise, France) with or without $0.05 \mathrm{IU} / \mathrm{mL}$ heparin. Four clones were isolated: three producing Abs (1C12, 1E12, and 2E1) that equally bound the PF4/H complex and PF4 alone (anti-PF4 Abs), and one that was mainly specific to $\mathrm{PF} 4 / \mathrm{H}$ (5B9). These Abs were isolated from ascitic fluid by using protein $\mathrm{G}$ columns (GE Healthcare). The murine anti-PF4/H Ab KKO was purchased from Thermo Fischer Scientific (Les Ulis, France).

\section{Human PF4-Specific Antibodies}

Human PF4-specific Abs were purified from patients as previously described, ${ }^{6}$ and classified into three groups according to their variable ability to activate platelets: group-1 Abs did not activate platelets (negative heparininduced platelet activation test [HIPA]), group-2 Abs induced positive HIPA results but only in the presence of heparin and were considered as typical HIT Abs, whereas group-3 Abs activated platelets even in the absence of heparin and were therefore defined as "autoantibodies."

\section{ELISA for Monoclonal PF4-Specific Antibodies}

The reactivity of purified KKO, 5B9, 1E12, 1C12, and 2E1 against $\mathrm{PF} 4 / \mathrm{H}$ complexes was evaluated by using the commercial IgG-specific ELISA kit Asserachrom HPIA IgG (Stago, Asnières, France) according to the manufacturer's recommendations, except that we used a peroxidase-conjugated goat anti-mouse IgG light chain $\mathrm{Ab}$ as a secondary $\mathrm{Ab}$ (Jackson ImmunoResearch, Ely, United Kingdom). Binding of these Abs to PF4 alone and to protamine sulfate or protamine sulfate $/ \mathrm{H}$ complexes was also assessed as previously described, ${ }^{11}$ using the peroxidase-conjugated goat anti-mouse IgG light chain Ab.

\section{ELISA for Human PF4-Specific Antibodies}

PF4/H complexes were preformed by incubating a mixture of $20 \mu \mathrm{g} / \mathrm{mL}$ PF4 and $0.5 \mathrm{IU} / \mathrm{mL}$ heparin (Heparin-Natrium25,000, Ratiopharm GmbH, Ulm, Germany) in PBS for 1 hour at room temperature (RT) and immobilized on a microtiter plate overnight at $4^{\circ} \mathrm{C} .^{12}$ Then, purified human Abs at different concentrations were incubated with $\mathrm{PF} 4 / \mathrm{H}$ complexes for 1 hour at RT. After washing $(150 \mathrm{mM} \mathrm{NaCl}, 0.1 \%$ Tween 20, pH 7.5), wells were incubated with peroxidase-conjugated goat anti-hIgG (1:20,000, Dianova, Hamburg, Germany) for 1 hour at RT. Bound Abs were subsequently detected by measuring the optical density (OD) at a wavelength of $450 \mathrm{~nm}$.

\section{Platelet Activation and Aggregation Tests}

Whole blood from healthy donors was collected in acidcitrate-dextrose supplemented with prostaglandin E1 (0.1 mM, Sigma-Aldrich, Saint-Quentin-Fallavier, France). ${ }^{13}$ Platelets were washed and suspended at a final count adjusted to $300 \times 10^{6} / \mathrm{mL}$. Serotonin release assay was performed as described, ${ }^{7}$ with different concentrations of KКО, 5B9, $1 \mathrm{E} 12,1 \mathrm{C} 12$, or 2E1, with or without UFH (Heparin Choay, Sanofi-Aventis, Gentilly, France). For HIPA assay, $75 \mu \mathrm{L}$ of washed platelets was incubated with $20 \mu \mathrm{L}$ purified Abs groups $1-3$ of $50 \mu \mathrm{g} / \mathrm{mL}$ with the low-molecular-weight heparin (reviparin) 0.2 aFXau. $^{14} \mathrm{~A}$ high heparin concentration $(100 \mathrm{IU} / \mathrm{mL})$ was added to inhibit platelet aggregation as a control. 


\section{SMFS Experiments}

Single-molecule force spectroscopy (SMFS) experiments were performed as previously described. ${ }^{6}$ To immobilize Abs on an atomic force microscope (AFM) tip, gold-coated silicon nitride cantilevers with a nominal spring constant of 6 and $30 \mathrm{pN} / \mathrm{nm}$ (Olympus Biolever, Tokyo, Japan) were coated with thiol-PEG$\mathrm{COOH}$ (Mw 3,400 Da; Nanocs, United States) and the - $\mathrm{COOH}$ group at the end of PEG linkers were activated with the amine coupling kit (Biacore, Uppsala, Sweden), containing the mixture of $0.4 \mathrm{M}$ 1-ethyl-3-(3-dimethylaminopropyl) carbodiimide hydrochloride (EDC) and $0.1 \mathrm{M} \mathrm{N}$-hydroxysuccinimide (NHS), for binding of proteins. ${ }^{15,16}$ The cantilevers were coated with human or monoclonal PF4-specific Abs at a concentration of $70 \mu \mathrm{g} / \mathrm{mL}$, and incubated for 30 minutes at RT and kept overnight at $4^{\circ} \mathrm{C}$. Free activated groups on the surfaces were blocked by adding $1.0 \mathrm{M}$ ethanolamine (Biacore, Uppsala, Sweden) for 1 hour at RT.

For immobilization of PF4 $(20 \mu \mathrm{g} / \mathrm{mL})$ or PF4/H complexes on the substrate, the same protocol as described for coating Abs on the AFM tip was used. PF4/H complexes were preformed by incubating a mixture of $20 \mu \mathrm{g} / \mathrm{mL}$ PF4 and $0.5 \mathrm{IU} / \mathrm{mL}$ heparin (Heparin-Natrium-25,000, Ratiopharm GmbH, Germany) in PBS for 1 hour at RT. ${ }^{17}$ For immobilization, proteins were kept on the PEG-coated Au surfaces overnight at $4^{\circ} \mathrm{C}$.

The measurements were performed in PBS using JPK NanoWizard 3 (Berlin, Germany) with a setpoint of $200 \mathrm{pN}$ and $\sim 1,000$ force-distance $(\mathbf{F}-\mathrm{D})$ curves per condition at a velocity of $1 \mu \mathrm{m} / \mathrm{s}^{18}$ Thermal off-rates (dissociation rates) of the bonds between Abs and PF4/H complexes were determined by applying the Bell-Evans model to the relation between loading rates and rupture forces, which were measured at tip speeds ranging 0.05 to $15 \mu \mathrm{m} / \mathrm{s}$ with $\mathrm{k}_{\text {effect }} \sim$ $\mathrm{k}_{\text {cantilever }}{ }^{19,20}$ The model describes that the rupture force increases proportionally to the natural logarithm of the loading rate during retraction. JPK data processing software (version $4.4 .18+$ ) was used to analyze the measured adhesion forces. The mean rupture force values and their corresponding errors were determined by applying Gaussian fits to the data using Origin software (version 9.1).

\section{Isothermal Titration Calorimetry}

Isothermal titration calorimetry (ITC) measurements were performed using a MicroCal iTC200 calorimeter (Malvern Instruments Ltd., Malvern, United Kingdom). PF4 in PBS 17 $\mu \mathrm{M}$ was added to the sample cell and a solution of moAbs (1.7 $\mu \mathrm{M})$ or human Abs (62 $\mathrm{nM})$ was loaded into the injection syringe. The $\mathrm{Ab}$ solution in the syringe was added by 19 injections of $2 \mu \mathrm{L}$ each into PF4 in the cell at $25^{\circ} \mathrm{C}$, at $1,000 \mathrm{rpm}$ stirring with 240 -second intervals. The one set of sites model was used to fit the binding isotherm data with nonlinear regression to determine the change in enthalpy $(\Delta H)$ of the interactions. The average and standard deviation were obtained from fitting ITC results.

\section{Dynamic Light Scattering}

To form complexes of PF4 with Abs, PF4 (20 $\mu \mathrm{g} / \mathrm{mL}$ final) was incubated with PF4-specific Abs ( $10 \mu \mathrm{g} / \mathrm{mL}$ final) at RT for 30 minutes before measurements. A dynamic light scattering
(DLS)-fixed scattering angle Zetasizer Nano-S system (Malvern Instruments Ltd.) and disposal cuvettes were used. The Z-average size, i.e., the hydrodynamic diameter, of PF4, Abs, or their complexes was measured in PBS at $25^{\circ} \mathrm{C}$ and light scattering was detected at $173^{\circ}$ using 10 repeating measurements. Data analysis was performed using the Zetasizer software, version 7.11 (Malvern Instruments Ltd.) and Origin software (version 9.1). DLS was also used to determine the zeta potential of the different PF4-specific Abs. Abs were diluted in water for zeta potential measurements, which were recorded in a folded capillary zeta cell at $25^{\circ} \mathrm{C}$ with six repetitions.

\section{Docking Analysis of Monoclonal Antibody Binding to PF4 Complexes}

$1 \mathrm{E} 12,1 \mathrm{C} 12$, and 2E1 cDNAs were sequenced by using the Sanger method, and a model of the three-dimensional (3D) structure of these Abs was then built by using MODELLER. ${ }^{21}$ The structures used as templates for homology modeling were PDB:5MYO for the variable heavy $(\mathrm{VH})$ chain and constant domains and PDB:4GMS for the variable light (VL) chain. The resulting 3D model was docked on PF4 (PDB:1F9Q), ${ }^{22}$ by using the MAbTope method ${ }^{21,23}$ optimized for $\mathrm{Ab} /$ antigen complexes. Structures were imaged by using the PyMOL Graphics System (v1.8). The prediction of the epitopes was based on the 30 best docking poses. Residues that were present at the interface in more than 20 poses had a very high probability of belonging to the interface, those present in 15 to 19 poses, 10 to 14 poses, and 5 to 9 poses had high, medium, and low probability, respectively, to be part of the interface.

\section{Ethics}

The use of human sera obtained from healthy volunteers and patients with HIT including the informed consent procedure was approved by the ethics board at the University Medicine Greifswald. All volunteers gave informed consent; all studies were performed according to the Declaration of Helsinki.

\section{Results}

\section{Antibody Binding to PF4/H Complexes and Platelet Activation Properties}

The binding to PF4/H complexes of all moAbs, KKO, 5B9, $1 \mathrm{C} 12,1 \mathrm{E} 12$, and 2E1 was dose-dependent as evidenced in ELISA. With 1C12, 1E12, and 2E1, the OD values were high, almost maximal at a low concentration of Abs $(0.5 \mu \mathrm{g} / \mathrm{mL})$, and their reactivity with $\mathrm{PF} 4 / \mathrm{H}$ complexes was very similar to that of KKO. In contrast, the OD value was lower with 5B9, with a maximum value measured with $5 \mu \mathrm{g} / \mathrm{mL}$, suggesting a lower affinity of 5B9 for PF4/H complexes (-Fig. 1A).

Human Abs, either non-platelet activating (group-1; nonpathogenic) or from typical HIT (group-2) or autoimmune HIT (group-3) patients, also displayed dose-dependent binding to PF4/H complexes as evidenced in ELISA ( - Fig. 1B). The highest $\mathrm{OD}_{450 \mathrm{~nm}}$ values were obtained with sera containing group-3 Abs, followed by group- 2 and group- 1 Abs. Binding patterns in ELISA of human group- 3 Abs resembled the 

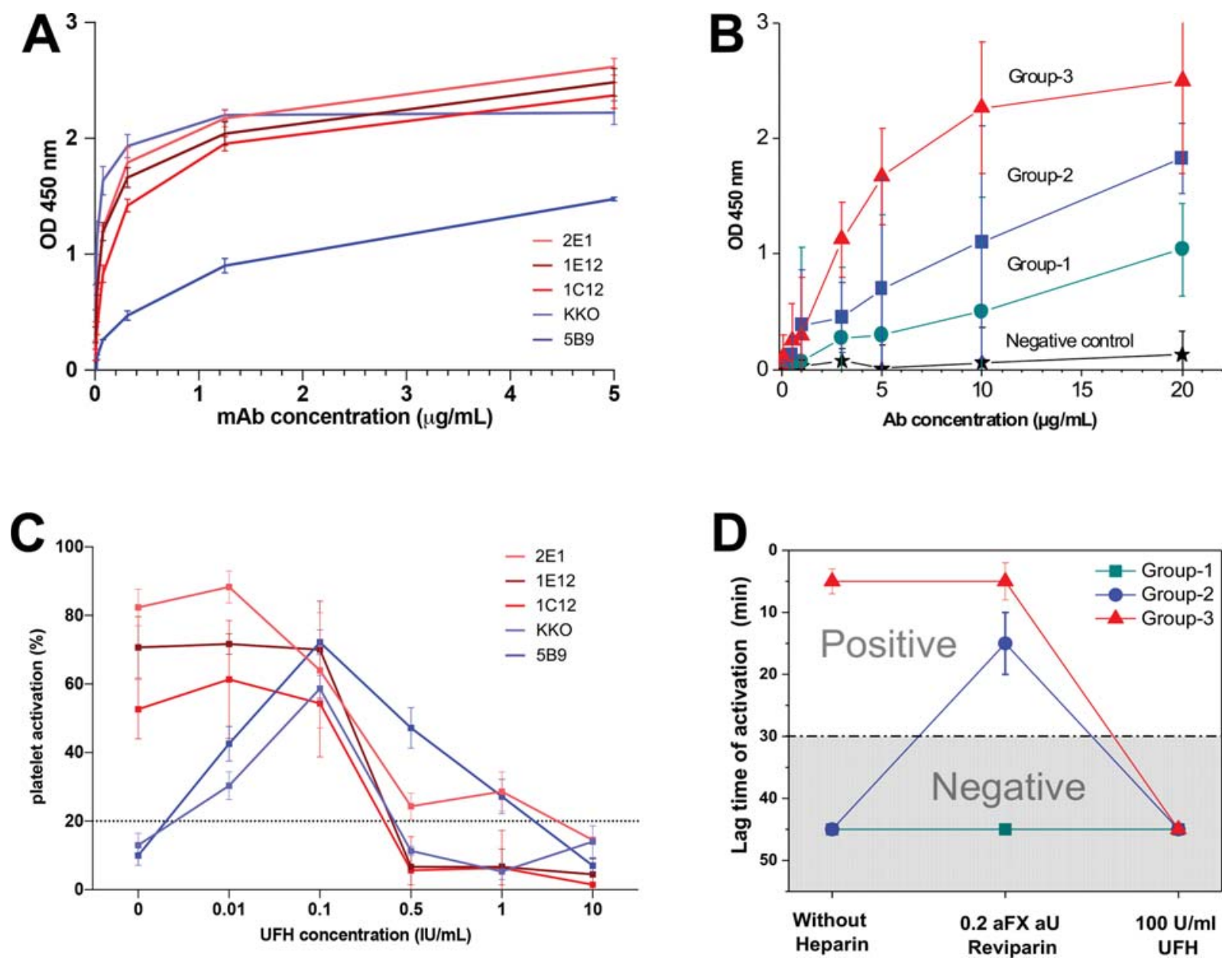

Fig. 1 Binding to PF4/H complexes and platelet activation properties of murine monoclonal and purified human PF4-specific IgG antibodies. Mean OD values \pm SEM obtained after incubation with coated PF4/H complexes of variable concentrations of (A) monoclonal PF4-specific antibodies KKO, 5B9, 1E12, 1C12, 2 E1 ( $n=3$ experiments) and (B) human PF4-specific Abs (groups 1-3) purified from five human sera for each group. Absorbances were measured by different enzyme immunoassays and photometers and OD values are therefore not directly comparable. (C) Serotonin release assay performed with 2E1, 1E12, and 1C12 in comparison with $5 \mathrm{~B} 9$ and KKO, with and without unfractionated heparin. Data are mean $(\%) \pm$ SEM of results obtained with three different platelet donors for each monoclonal antibody. (D) HIPA performed with affinitypurified PF4-specific IgG from patients of groups 1-3. The low-molecular-weight heparin reviparin was used in the HIPA test at low concentration ( $0.2 \mathrm{aFXaU}) ; n=5$ donors tested for each antibody group. Data are given as lag time until platelet activation ( \pm SEM); as shorter the lag time the stronger the reactivity; below dashed lines are HIPA negative. Human data in (B) and (D) were partially adapted from $\mathrm{Nguyen}$ et al. ${ }^{6} \mathrm{IgG}$, immunoglobulin G; OD, optical density; PF4, platelet factor 4; SEM, standard error of mean.

pattern found with 1C12, 1E12, 2E1, and KKO, while group-2 Abs exhibited a binding pattern similar to the ones found with 5 B9.

In the presence of low concentrations of UFH, KKO and 5B9 induced strong platelet activation (-Fig. 1C) while no activation was observed in the absence of heparin. This platelet activation pattern is identical to that obtained with typical group-2 HIT Abs (-Fig. 1D). In contrast, 1C12, $1 \mathrm{E} 12$, and $2 \mathrm{E} 1$ activated platelets without the addition of heparin (-Fig. 1C), similarly to group-3 Abs from autoimmune HIT patients ( - Fig. 1D). High concentrations of heparin inhibited platelet activation induced by all monoclonal and human Abs tested.

\section{Antibody Binding Strength and Kinetics of Interaction with PF4/H Complexes}

The binding strength and kinetics of interaction of moAbs and human Abs with PF4/H complexes were analyzed by
SMFS (-Fig. 2). Single IgG molecules of each Ab type were coated to a distinct AFM-cantilever tip and brought into contact with the surface coated with PF4/H complexes for measuring their binding strength/force $\mathbf{F}$ (-Fig. 2A, B). Representative histograms of binding strength $\mathbf{F}$ measured with the moAbs (-Fig. 2C) and human PF4-specific Abs ( - Fig. 2D) allowed the determination of mean interaction forces. Only some nonspecific interactions were recorded with controls, including mouse IgG2b (mIgG2b) and hIgG purified from healthy donors. When moAbs were tested, the interaction forces measured were lower with KKO $(42.0 \pm 0.4 \mathrm{pN})$ than with $5 \mathrm{~B} 9(67.9 \pm 1.8 \mathrm{pN})$, while the highest values were obtained with $1 \mathrm{C} 12(77.7 \pm 0.7 \mathrm{pN})$ and $1 \mathrm{E} 12(76.6 \pm 0.9 \mathrm{pN})$. Notably, 2E1 exhibited a different reactivity pattern with $\mathrm{PF} 4 / \mathrm{H}$, i.e., yielding two different groups of interaction force values, at $64.1 \pm 1.8 \mathrm{pN}$ and $139.5 \pm 2.0 \mathrm{pN}$, respectively. These data with moAbs were compared with those obtained with the three different 

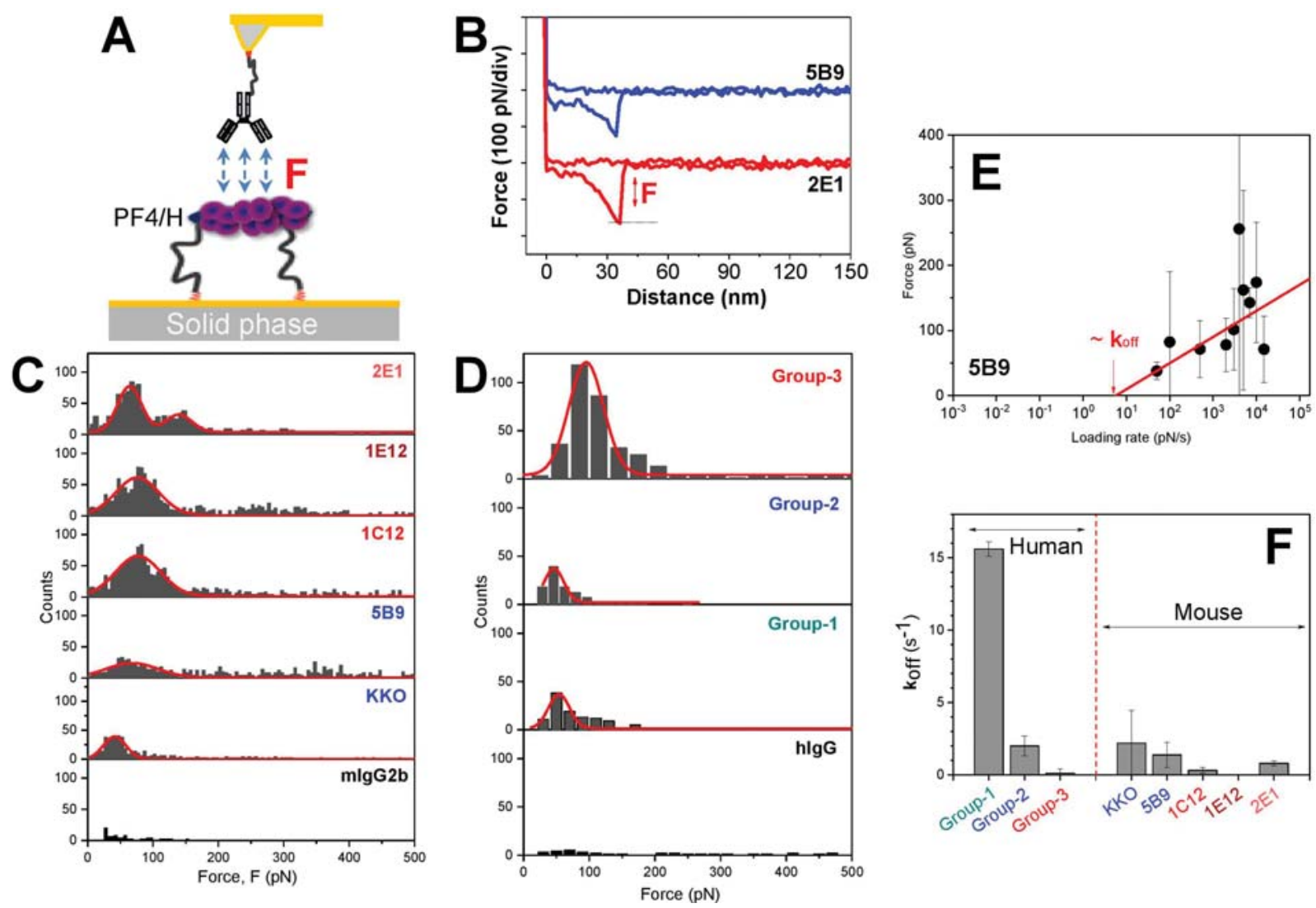

Fig. 2 Binding strength of PF4-specific moAbs and human Abs to PF4/H complexes. (A) A single antibody was attached covalently on an AFM-tip via PEG linkers, and PF4/H complexes were immobilized on the substrate for measurement of their interaction force $F$. (B) Example of forcedistance curves showing force $F$ obtained with $5 B 9$ and 2E1. (C, D) Rupture forces and corresponding standard errors determined by a Gaussian fit (solid curves) with (C) moAbs KKO, 5B9, 2E1, 1E12, 1C12, and mlgG2b, or (D) human Abs of groups 1-3 and human control IgG (hlgG). (E) Representative rupture forces recorded for $5 \mathrm{~B} 9$ at different loading rates allowed the determination of the thermal off-rate ( $k_{\text {off }}$ ) of the interaction. (F) Average $k_{\text {off }}$ values and standard deviations were obtained with moAbs and human Abs by fitting forces measured at different loading rates. Small koff value is not visible for $1 \mathrm{E} 12$ in (F). Human data in (D) and (F) were adapted from Nguyen et al. ${ }^{6}$ Note: low $k_{\text {off }}=$ stable binding. AFM, atomic force microscope; IgG, immunoglobulin G; mlgG2b, mouse control IgG; PF4, platelet factor 4.

groups of human Abs (-Fig. 2D). ${ }^{6}$ The interactions forces measured with different sera were relatively weak with group-1 Abs $(46.3 \pm 8.1 \mathrm{pN})$, stronger with group-2 Abs $(60.6 \pm 15.4 \mathrm{pN})$, and the highest values were recorded with group-3 Abs $(72.4 \pm 26.2 \mathrm{pN}){ }^{6}$

To further understand the binding kinetics of reactions, we determined thermal off-rates $\left(k_{\text {off }}\right)$ of the interactions between Abs and PF4/H complexes. For this, binding forces were recorded at velocities ranging 50 to $15,000 \mathrm{~nm} / \mathrm{s}$ for all $\mathrm{mAb}$ types. By analyzing binding forces at different loading rates and applying the Bell-Evans model, ${ }^{19,20}$ we found different thermal off-rates $\left(k_{\text {off }}\right)$ among $\mathrm{Ab}$ types (-Fig. 2F). As an example, data for 5B9 are shown in -Fig. 2E. For other Abs, rupture forces at different loading rates are given in the Supplementary Material (-Supplementary Fig. S1 [available in the online version]). No $k_{\text {off }}$ value as high as that of group-1 human Abs was obtained with any of the moAbs tested. The $k_{\text {off }}$ values measured with KKO or 5B9 were comparable to that of group-2 Abs, and slightly lower values were recorded for $1 \mathrm{C} 12$ and 2E1. The lowest $k_{\text {off }}$ (indicating the most stable binding) was obtained for $1 \mathrm{E} 12$, and group-3 Abs.

\section{Antibody Binding to PF4 on a Solid Phase and in Solution}

The binding properties of the five moAbs on PF4 immobilized on a solid phase were also assessed by SMFS. Each Ab was linked to an AFM tip while PF4 molecules were immobilized on the substrate, and interaction forces were compared with those obtained with the three groups of human PF4-specific Abs. As expected, the two anti-PF4/H moAbs, 5B9 and KKO, exhibited non-significant interactions with PF4 alone, comparable to control murine IgG2b. In contrast, 2E1, 1E12, and 1C12 clearly bound to PF4 (-Fig. 3A), but to a lower extent than human group-3 Abs (-Fig. 3B).

To evaluate the interactions of Abs with PF4 in solution, ITC experiments were performed. Very low heat release $(\Delta H)$ was measured when control $\operatorname{IgG} 2 \mathrm{~b}$, KKO, or $5 \mathrm{~B} 9$ interacted with PF4 alone ( $\mathbf{F i g . 3 C - E}$ ), and values obtained were similar when group-2 Abs were tested (-Fig. 3j). In contrast, the heat released from the interaction of either 1E12 or 1C12 with PF4 (- Fig. 3F-G) was higher and similar to the one obtained with human group-3 Abs (-Fig. 3K). On the other hand, a different pattern was recorded with 2E1 

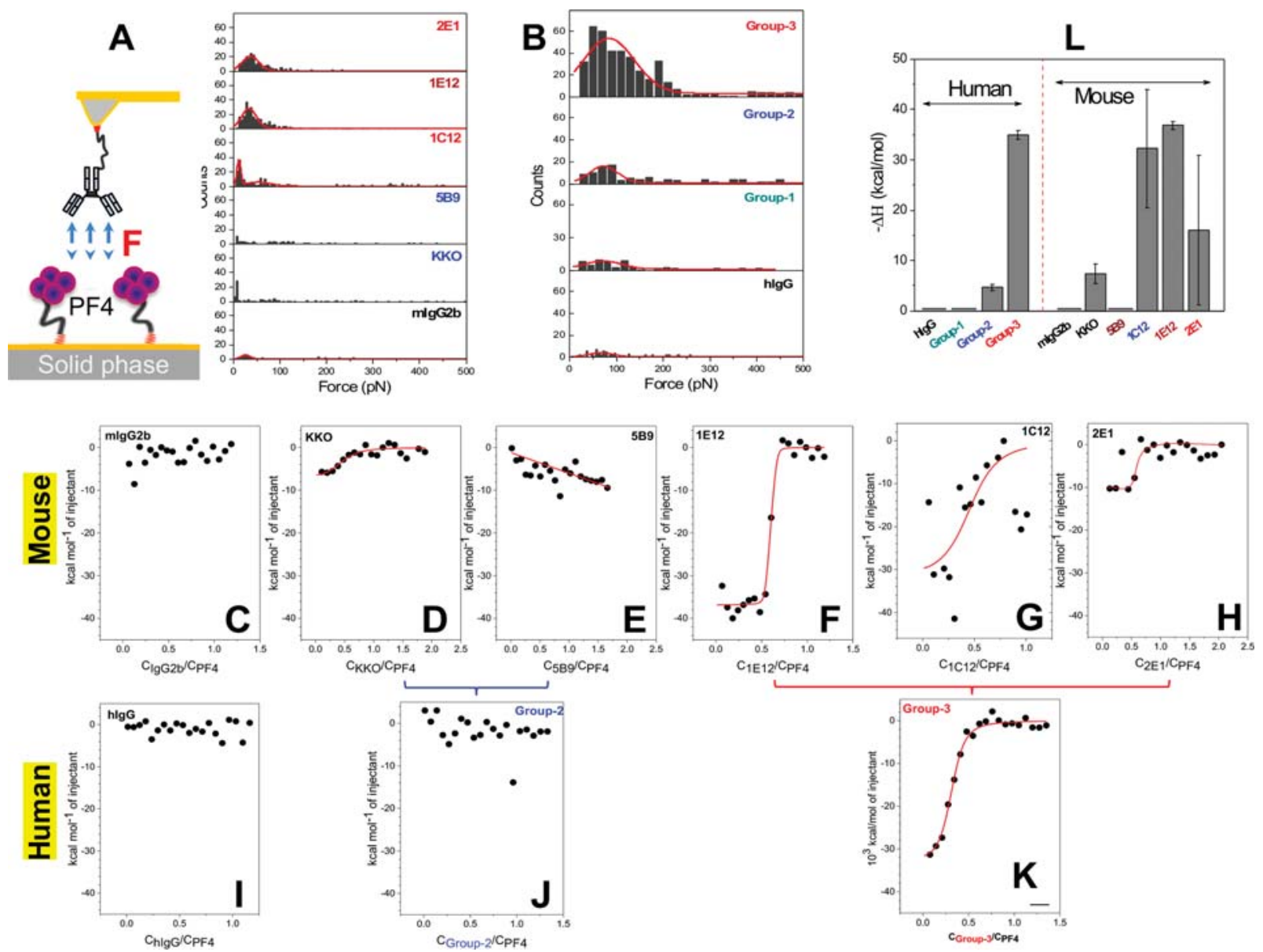

Fig. 3 Binding of monoclonal and human IgG Abs to PF4 in SMFS and ITC. (A) Binding strength of moAbs (left panel) and (B) polyclonal human Abs (right panel) to PF4 alone measured by SMFS (inset, A). (C-K) Representative patterns of integrated heat released by interactions of different Abs with PF4 measured by ITC. (L) Summary data of mean \pm SD resulted from fitting ITC data $(n=2$ experiments for $1 E 12$, and 1 for other moAbs). Human data in (B), (L), (J), and (K) were adapted from Nguyen et al. ${ }^{6} \mathrm{IgG}$, immunoglobulin G; ITC, isothermal titration calorimetry; PF4, platelet factor 4; SD, standard deviation; SMFS, single-molecule force spectroscopy.
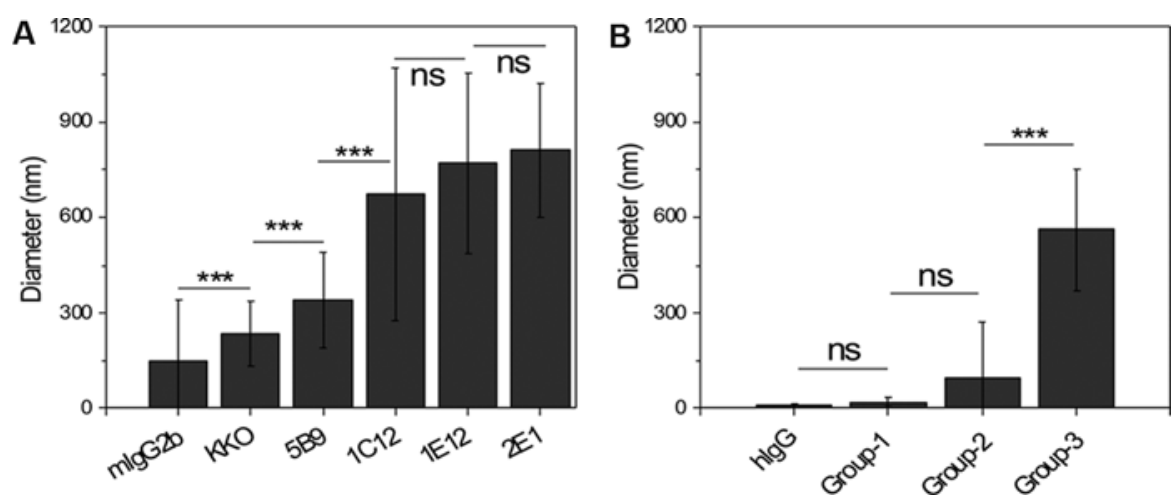

Fig. 4 Oligomerization of PF4 induced by monoclonal and human Abs assessed by DLS. Size of PF4-IgG complexes with (A) moAbs and (B) human Abs. Data are mean diameter $(\mathrm{nm}) \pm \mathrm{SD}$ from three repetitions. The statistic was taken via one-way ANOVA test: $\mathrm{ns}=\mathrm{no}$ significant difference $(p>0.01) ;{ }^{* * *}=$ significant difference $(p<0.01)$. Human data in (B) were adapted from Nguyen et al. ${ }^{6}$ ANOVA, analysis of variance; DLS, dynamic light scattering; PF4, platelet factor 4; SD, standard deviation.

(-Fig. 3H), as it released much lower heat than $1 \mathrm{E} 12$ and 1C12 when interacting with PF4 (-Fig. 3F-H). These data suggested that $1 \mathrm{C} 12,1 \mathrm{E} 12$ (and to a lesser extent 2E1), as well as human group-3 Abs can cluster PF4 molecules and that this capacity is absent or very low for $5 \mathrm{B9}, \mathrm{KKO}$, and group-2 Abs.
By DLS experiments, we also assessed the size of complexes formed in solution between PF4 and moAbs (-Fig. 4A) or human Abs (-Fig. 4B). As expected, complexes formed with $1 \mathrm{E} 12,1 \mathrm{C} 12,2 \mathrm{E} 1$, were relatively large and similar to those formed with human group-3 Abs, whereas $\mathrm{KKO}, 5 \mathrm{~B} 9$, and group 2 Abs produced much smaller complexes with PF4 (-Fig. 4). 

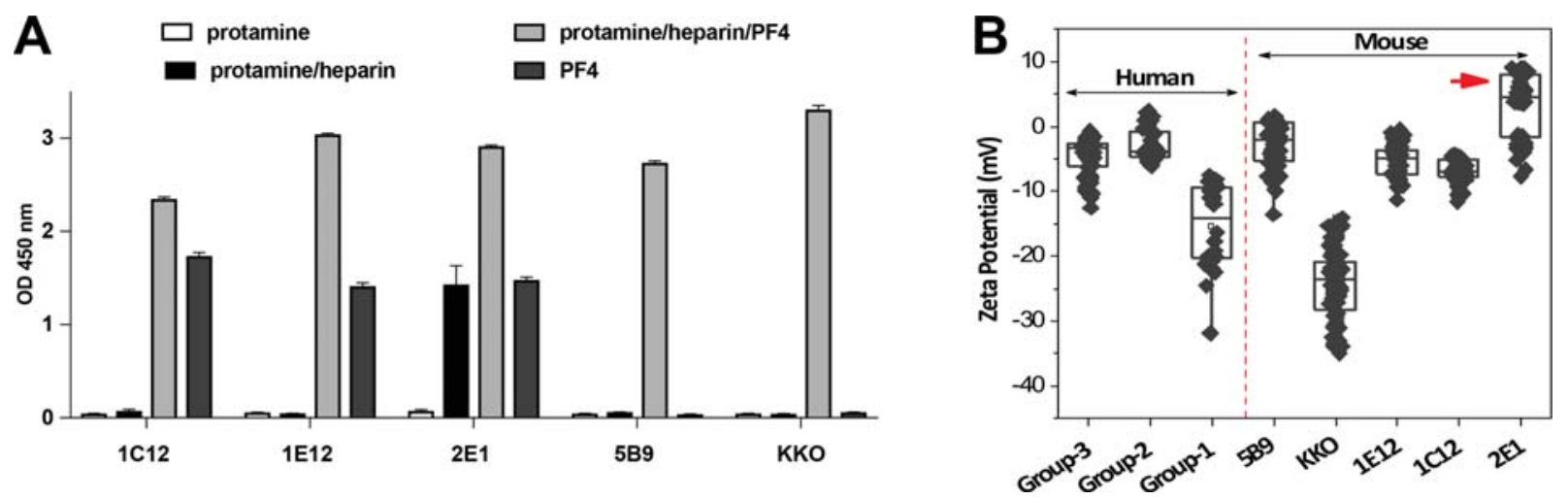

Fig. 5 Binding specificity and surface charge of PF4-specific monoclonal Abs. (A) Binding of PF4-specific moAbs to protamine, heparin, and PF4 complexes. OD values obtained after incubation of $5 \mathrm{~B} 9, \mathrm{KKO}(10 \mu \mathrm{g} / \mathrm{mL}), 1 \mathrm{C} 12,1 \mathrm{E} 12$, and $2 \mathrm{E} 1(0.5 \mu \mathrm{g} / \mathrm{mL})$ in wells coated with protamine (white), protamine/heparin (black), protamine/heparin/PF4 (light grey) and PF4 alone (dark grey). (B) Zeta potential of human and monoclonal PF4-specific IgG Abs measured in DLS $(n=3-4)$. DLS, dynamic light scattering; OD, optical density; PF4, platelet factor 4.

\section{Charge-Related Interactions of Monoclonal PF4- Specific Abs}

We further evaluated by ELISA the binding of each moAb to PF4 alone, to protamine alone, and to heparin immobilized by protamine, in the presence or absence of PF4. High OD values were measured with $\mathrm{KKO}, 5 \mathrm{~B} 9,1 \mathrm{C} 12,1 \mathrm{E} 12$, and 2E1 in the presence of PF4/H complexes, and only 1C12, 1E12, and 2E1 interacted with PF4 alone (- Fig. 5A). Surprisingly, significant binding of $2 \mathrm{E} 1$ to heparin immobilized by protamine in the absence of PF4 was also measured, without any interaction with protamine alone. This supported that this $\mathrm{Ab}$, apart from binding to PF4, also interacted with heparin alone. Next, we determined the zeta potential of all Abs, which was either neutral (5B9 and human group-3 Abs), slightly negatively charged (1E12, 1C12, and human group-2 Abs), or strongly negatively charged (KKO and human group-1 Abs). A positive zeta potential was only found for 2E1 ( - Fig. 5B).

\section{Prediction of Antibody Binding Sites on PF4 by a Docking Model}

The binding sites of 1C12,1E12, and 2E1 on PF4 were predicted using the MAbTope method, based on the VH and VL sequences of each moAb and the crystal structure of the PF4 tetramer. The results were then compared with those obtained previously with $5 \mathrm{~B} 9^{10}$ and by crystallography with KKO. ${ }^{23}$ According to our model, 1C12,1E12, and 2E1 appear to bind to monomers B, C, and D (-Fig. 6) and to interact with 23, 31, and $21 \mathrm{AA}$ residues, respectively. It is noteworthy that 15 amino acid residues $(6,4$, and 5 in monomers $B, C$, and $D$, respectively) are likely involved in the binding of the three anti-PF4 moAbs, including 8,5 , and 8 with a very high or high probability for $1 \mathrm{C} 12,1 \mathrm{E} 12$, and 2E1, respectively. Six additional residues also potentially contribute to the binding site, each interacting with variable combinations of two Abs. Eleven other amino acids have also been identified to interact exclusively with $1 \mathrm{E} 12$, and only two or five with $2 \mathrm{E} 1$ and $1 \mathrm{C} 12$, respectively.

\section{Discussion}

In this study, we analyzed several moAbs, which bind to PF4 and activate platelets (5B9, KKO, 1C12, 1E12, and 2E1), and compared their characteristics with those of human Abs affinity-purified from sera of patients with typical HIT or autoimmune HIT.

The three new monoclonal IgG Abs with a human Fc fragment, 1C12, 1E12, and 2E1, clearly differ in terms of specificity and functional activity from the monoclonal antiPF4/H IgG Abs, KKO, and 5B9. ${ }^{8,10}$ Indeed, 5B9 and KKO exhibit similar properties to typical heparin-dependent, plateletactivating human HIT Abs (group-2), whereas 1C12, 1E12, and $2 \mathrm{E} 1$ behave similarly to human Abs of patients with “autoimmune" HIT (group-3).

$1 \mathrm{C} 12,1 \mathrm{E} 12,2 \mathrm{E} 1$, and group-3 Abs displayed a very high affinity for $\mathrm{PF} 4 / \mathrm{H}$ complexes in comparison with 5B9, as suggested by the 10 times lower concentrations necessary for reaching maximum OD values in ELISA. These Abs, therefore, resemble human group-3 Abs, whose OD values were systematically higher compared with the ones obtained with human group-2 Abs. The higher affinity of 1C12, 1E12, and $2 \mathrm{E} 1$ for $\mathrm{PF} 4 / \mathrm{H}$ complexes was also confirmed by SMFS measuring strong interaction forces of these Abs with $\mathrm{PF} 4 / \mathrm{H}$ complexes, similarly to those recorded with human group-3 Abs. In contrast, interaction forces of 5B9 and KKO with PF4/ $\mathrm{H}$ complexes were lower and close to those measured with human group-2 Abs. The high OD values obtained in ELISA with KKO, despite interaction forces with $\mathrm{PF} 4 / \mathrm{H}$ complexes were lower than the ones measured with $5 \mathrm{~B} 9$, can be explained by a more stable binding of KKO to PF4/H (with a higher count number). Our data thus illustrate that absorbance values measured in PF4-specific ELISA are always resulting from a variable composite of Ab affinity and avidity. High-affinity binding of anti-PF4 Abs to PF4 complexed with polyanions other than heparin may also contribute to the pathogenic effects of such Abs in patients with autoimmune HIT. To that extent, further studies may be useful to demonstrate that $1 \mathrm{C} 12,1 \mathrm{E} 12$, and $2 \mathrm{E} 1$ can bind not only to PF4 complexed with glycosaminoglycans (GAGs) exposed on cell surface, such as chondroitin sulfate and heparan sulfate, but also to PF4 in complex with polyphosphates, or DNA/RNA.

$1 \mathrm{C} 12,1 \mathrm{E} 12$, and 2E1, similarly to human group-3 Abs associated with autoimmune HIT, also bound to PF4 alone as shown by ELISA, SMFS, ITC, and DLS. In contrast, 5B9, KKO, and 


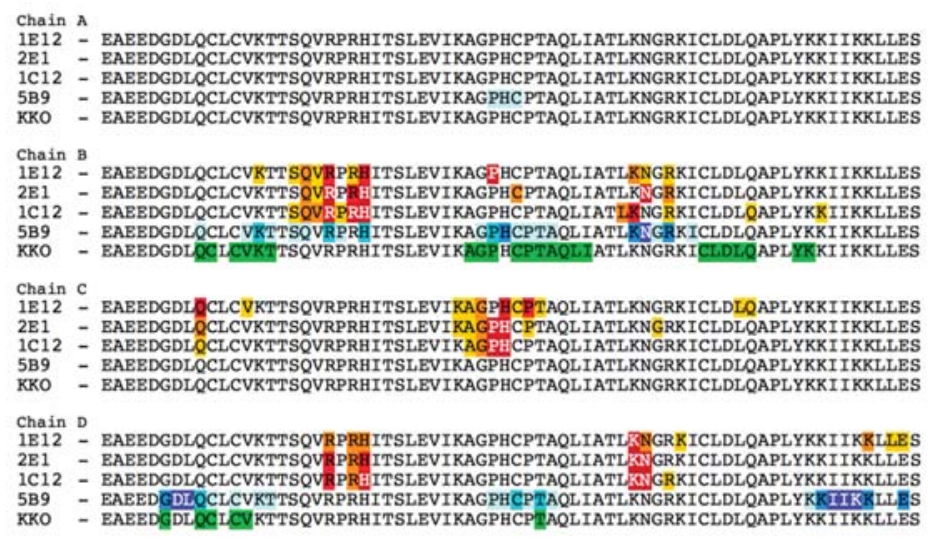

B

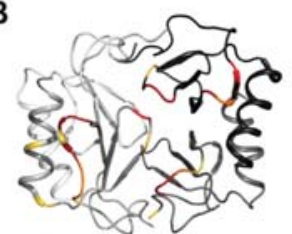

$1 \mathrm{C} 12$

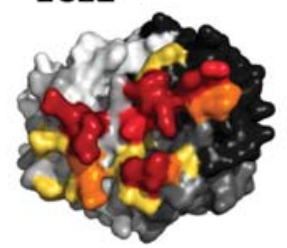

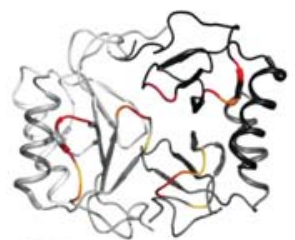

2E1

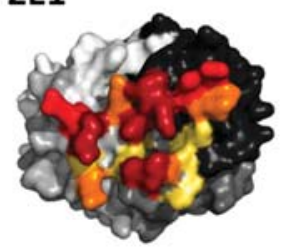

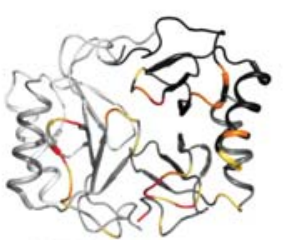

1E12

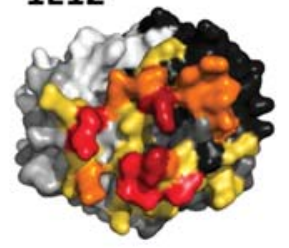

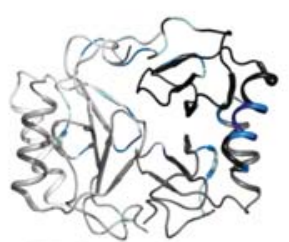

5B9

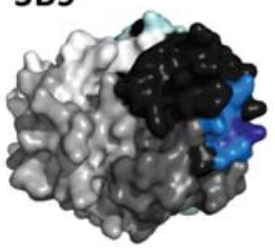

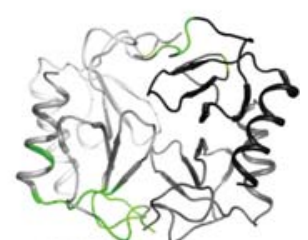

КKO

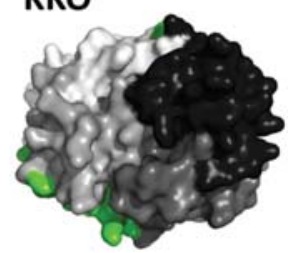

Fig. 6 Epitope mapping of antibody-PF4 interactions of 2E1, 1C12, and 1E12 compared with 5B9 and KKO. Residues of PF4 monomers (A) are colored according to their probability of contributing to the epitopes recognized by moAbs, as given by MAbTope. Colors for 1C12, 2E1, and 1E12 are dark red (very high probability), red (high probability), orange (medium probability), and yellow (low probability). Colors for $5 \mathrm{~B} 9$ are dark blue (very high probability), blue (high probability), cyan (medium probability), and light cyan (low probability). Residues of the KKO epitope are shown in green. (B) Surface and cartoon views of PF4 tetramer are shown in shades of gray (white: chain A, light gray: chain B, dark gray: chain C, black: chain D). PF4, platelet factor 4.

human group-2 Abs bound only to PF4/H complexes. Interestingly, Sachais et al had also found that KKO was able to induce oligomerization of PF4, ${ }^{24}$ unlike RTO, another PF4-specific Ab unable to induce any platelet activation. ${ }^{8}$ However, in our study only a small increase in aggregate size (measured by DLS) was evidenced when KKO, 5B9, and group 2 Abs were incubated with PF4, and no major changes in energy release were measured. In contrast, 1C12, 1E12, 2E1, or human group3 Abs formed larger aggregates in the presence of PF4 alone. This raises the interesting possibility that $1 \mathrm{C} 12,1 \mathrm{E} 12$, and 2E1 may facilitate on the platelet surface the formation of ultralarge and stable complexes of PF4, which are critical in the pathogenesis of HIT. ${ }^{25}$ Considering the symmetry of PF4 tetramers, and based on our docking analysis, one IgG may bind two distinct PF4 tetramers with its Fab arms, and two IgG may bind on two different monomers of the same PF4 tetramer (-Fig. 7). This process allows the formation of very large PF4 clusters on the cell surface and probably helps Fc parts of these Abs to be aligned in close proximity, allowing them to crosslink FcrRIIa receptors enough to induce strong signal transduction and platelet activation even in the absence of heparin. On the other hand, platelet activation was fully inhibited in the presence of high concentrations of heparin, confirming that dissociation of PF4 complexes from the platelet surface fully abrogated platelet activation induced by $1 \mathrm{C} 12,1 \mathrm{E} 12$, and $2 \mathrm{E} 1$.
It has recently been proposed that autoimmune HIT Abs can cluster PF4 and change its conformation, which allows binding of heparin-dependent Abs. ${ }^{6,26}$ Our findings are consistent with this hypothesis, but it remains unclear whether the same effects are caused by all three moAbs tested. This requires further studies, especially as we now know that the characteristics of typical or autoimmune HIT Abs may differ, like those of moAbs. Although the binding sites for 1C12, 1E12, and 2E1 on PF4 seem to be very close to each other, as predicted by our docking model, some differences were evidenced in SMFS and ITC experiments. The lowest $k_{\text {off }}$ value in SMFS was measured with $1 \mathrm{E} 12$, indicating that this $\mathrm{Ab}$ binds to $\mathrm{PF} 4 / \mathrm{H}$ complexes with the highest stability. Moreover, $1 \mathrm{E} 12$ induced the highest thermal energy release in ITC suggesting that this Ab can elicit major conformational changes of PF4. Of the three new moAbs assessed, the characteristics of 1E12 are closest to those of human autoimmune HIT group-3 Abs. ${ }^{6}$

Also, the moAb 2E1 is of interest, since the bimodal distribution of its interaction forces in SMFS suggests variable $\mathrm{Ab}$ binding modalities to $\mathrm{PF} / \mathrm{H}$ complexes. This is further supported by its high thermal-off rate $\left(k_{\text {off }}\right)$ combined with relatively low thermal energy release (compared with $1 \mathrm{E} 12$ or 1C12). The explanation is that this moAb binds to $\mathrm{PF} 4 / \mathrm{H}$ complexes via two mechanisms. The one is classic (but rather weak) Ab-antigen binding and the second is electrostatic 

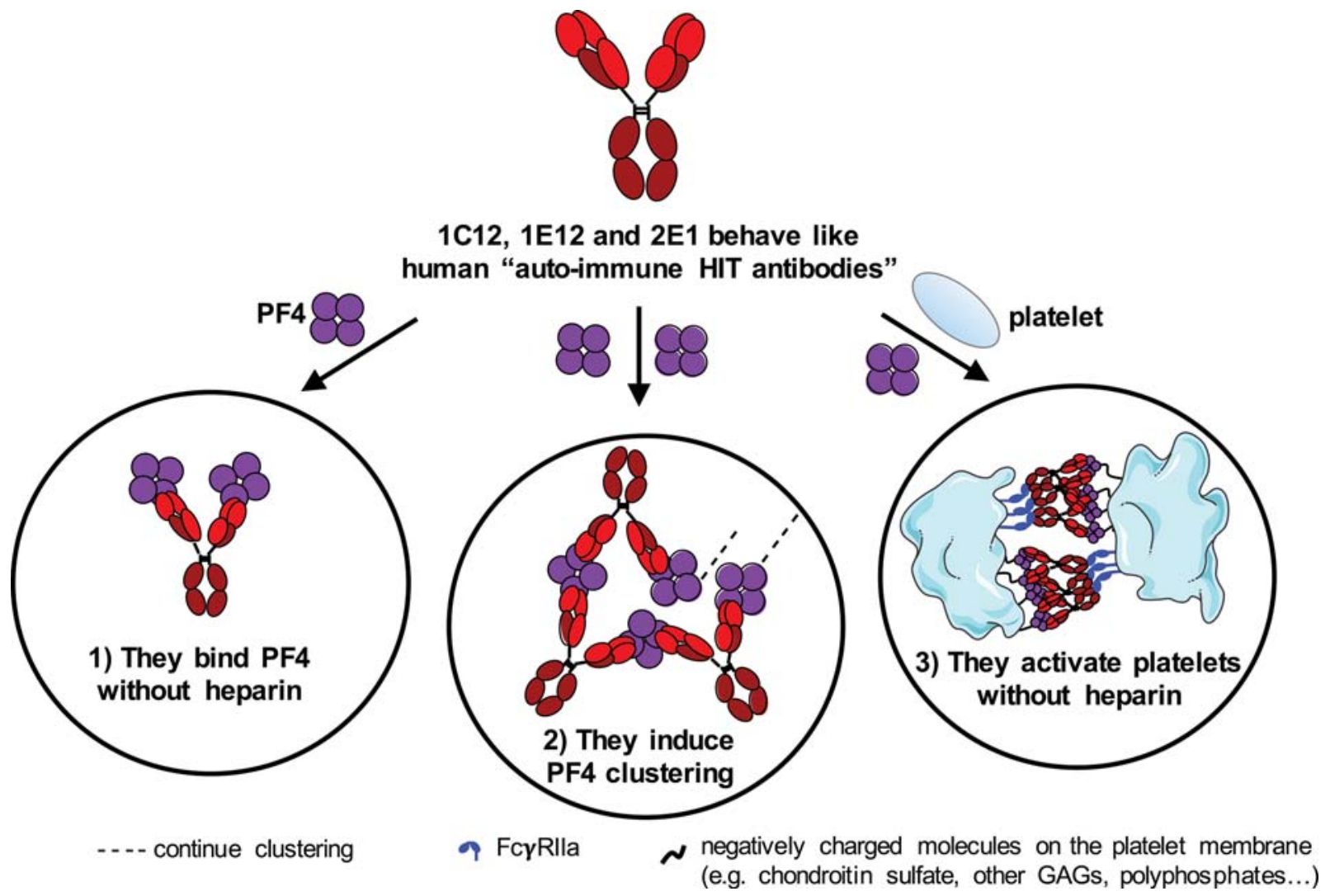

Fig. 7 Binding and functional properties of 1C12, 1E12, and 2E1 shared with human "autoimmune" HIT antibodies. HIT, heparin-induced thrombocytopenia.

interaction between a positively charged region of 2E1 (not yet identified) and heparin. Accordingly, the zeta potential of 2E1 showed the strongest positive charge of all Abs tested. These cooperative two binding mechanisms result in a high avidity of this $\mathrm{Ab}$ for $\mathrm{PF} 4 / \mathrm{H}$ complexes, and support its capacity to activate platelets.

The characteristics of our three new moAbs suggest that the reactivity patterns of Abs associated with autoimmune HIT can be attributed to at least two different mechanisms. One is strong $\mathrm{Ab}$ binding to PF4 inducing a conformational change with consecutive binding of other Abs, as shown with human HIT group- $3 \mathrm{Abs}^{6}{ }^{6}$ The other is weak Ab binding to PF4 via the antigen-binding site with additional charge-related interaction with heparin or other polyanions on the platelet surface like chondroitin sulfate or polyphosphates. ${ }^{27}$ These Abs cannot be differentiated in functional assays since they both strongly induce platelet activation independent of heparin via FcrRIIA, which, however, is inhibited by high concentrations of heparin. Further studies are needed to confirm the existence of such a dual binding pattern of patient-derived autoimmune HIT Abs.

For many years, we have known that the specificity and functional properties of IgG Abs to PF4 can be variable from one patient with HIT to another. In addition, several PF4specific IgG Abs with different characteristics may coexist in the same individual. ${ }^{6,28}$

$1 \mathrm{E} 12$ seems to have characteristics resembling those of human autoimmune HIT Abs most closely. 2E1 on the other hand indicates a potentially new mechanism of $\mathrm{Ab}$ binding to PF4(/polyanion) complexes. Together with KKO and 5B9, which behave like classical HIT Abs, 1E12, 1C12, and 2E1 may help to further investigate and understand mechanisms of HIT.

\section{What is known about this topic?}

- Heparin-induced thrombocytopenia (HIT) is caused in most patients by platelet-activating IgG antibodies (Abs) directed against complexes of platelet factor 4 bound to heparin $(\mathrm{PF} 4 / \mathrm{H})$.

- HIT can sometimes present as an autoimmune disease, with affected patients exhibiting platelet activation even without heparin, associated with the detection of both anti-PF4/H IgG and IgG Abs able to bind native PF4.

- Mechanistic studies with Abs from patients have limitations as they constitute a polyclonal mixture of Abs with different characteristics.

\section{What does this paper add?}

- 1C12, 1E12, and 2E1 are new monoclonal antibodies with a human Fc fragment and they mimic anti-PF4 antibodies present in autoimmune HIT.

- 2E1 displays a bivalent binding mechanism to PF4 via its antigen recognition site and charge-related interactions with heparin, which could also be found in humans.

- 1C12, 1E12, and 2E1 are useful tools for better understanding the atypical and severe presentation of autoimmune HIT. 


\section{Authors' Contributions}

C.V. and T.H.N. performed and designed the research, analyzed the data, and wrote the manuscript. J.R., A.G., and Y.G. designed the research, analyzed the data, and wrote the manuscript. C.P. analyzed the data and wrote the manuscript. N.C., A.P., and N.N. performed the research and analyzed the data. All authors reviewed and approved the final version of the manuscript.

\section{Funding}

This work was supported by the Deutsche Forschungsgemeinschaft (DFG, German Research Foundation (NG 133/ 1-2) and the Institut pour la Recherche sur la Thrombose et l'Hémostase (IRTH).

\section{Conflict of Interest}

None declared.

\section{Acknowledgments}

We thank B Cell Design for providing us the monoclonal antibodies 5B9, 1C12, 1E12, and 2E1. We also thank Ina Buchholz and Mihaela Delcea for their support with ITC experiments.

\section{References}

1 Greinacher A. Heparin-induced thrombocytopenia. N Engl J Med 2015;373(19):1883-1884

2 Kreimann M, Brandt S, Krauel K, et al. Binding of anti-platelet factor 4/heparin antibodies depends on the thermodynamics of conformational changes in platelet factor 4. Blood 2014;124(15): 2442-2449

3 Arepally GM, Ortel TL. Heparin-induced thrombocytopenia. Annu Rev Med 2010;61:77-90

4 Greinacher A, Selleng K, Warkentin TE. Autoimmune heparininduced thrombocytopenia. J Thromb Haemost 2017;15(11): 2099-2114

5 Padmanabhan A, Jones CG, Bougie DW, et al. Heparin-independent, PF4-dependent binding of HIT antibodies to platelets: implications for HIT pathogenesis. Blood 2015;125(01):155-161

6 Nguyen TH, Medvedev N, Delcea M, Greinacher A. Anti-platelet factor 4/polyanion antibodies mediate a new mechanism of autoimmunity. Nat Commun 2017;8:14945

7 Pouplard C, Amiral J, Borg JY, Vissac AM, Delahousse B, Gruel Y. Differences in specificity of heparin-dependent antibodies developed in heparin-induced thrombocytopenia and consequences on cross-reactivity with danaparoid sodium. Br J Haematol 1997; 99(02):273-280

8 Arepally GM, Kamei S, Park KS, et al. Characterization of a murine monoclonal antibody that mimics heparin-induced thrombocytopenia antibodies. Blood 2000;95(05):1533-1540

9 Nguyen TH, Greinacher A. Platelet factor 4/heparin complexes present epitopes differently on solid-phase vs platelet surfaces. Blood 2017;129(26):3498-3501

10 Kizlik-Masson C, Vayne C, McKenzie SE, et al. 5B9, a monoclonal antiplatelet factor 4/heparin IgG with a human Fc fragment that mimics heparin-induced thrombocytopenia antibodies. J Thromb Haemost 2017;15(10):2065-2075
11 Pouplard C, Leroux D, Rollin J, Amiral J, May MA, Gruel Y. Incidence of antibodies to protamine sulfate/heparin complexes incardiac surgery patients and impact on platelet activation and clinical outcome. Thromb Haemost 2013;109(06):1141-1147

12 Juhl D, Eichler P, Lubenow N, Strobel U, Wessel A, Greinacher A. Incidence and clinical significance of anti-PF4/heparin antibodies of the IgG, IgM, and IgA class in 755 consecutive patient samples referred for diagnostic testing for heparin-induced thrombocytopenia. Eur J Haematol 2006;76(05):420-426

13 Cattaneo M, Cerletti C, Harrison P, et al. Recommendations for the standardization of light transmission aggregometry: a consensus of the Working Party from the Platelet Physiology Subcommittee of SSC/ISTH. J Thromb Haemost 2013. Doi: 10.1111/jth.12231

14 Greinacher A, Amiral J, Dummel V, Vissac A, Kiefel V, MuellerEckhardt C. Laboratory diagnosis of heparin-associated thrombocytopenia and comparison of platelet aggregation test, heparin-induced platelet activation test, and platelet factor 4/heparin enzymelinked immunosorbent assay. Transfusion 1994;34(05):381-385

15 Nguyen TH, Greinacher A, Delcea M. Quantitative description of thermodynamic and kinetic properties of the platelet factor 4 / heparin bonds. Nanoscale 2015;7(22):10130-10139

16 Nguyen TH, Steinbock LJ, Butt HJ, Helm M, Berger R. Measuring single small molecule binding via rupture forces of a split aptamer. J Am Chem Soc 2011;133(07):2025-2027

17 Krauel K, Weber C, Brandt S, et al. Platelet factor 4 binding to lipid A of Gram-negative bacteria exposes PF4/heparin-like epitopes. Blood 2012;120(16):3345-3352

18 Merkel R, Nassoy P, Leung A, Ritchie K, Evans E. Energy landscapes of receptor-ligand bonds explored with dynamic force spectroscopy. Nature 1999;397(6714):50-53

19 Evans E. Probing the relation between force-lifetime-and chemistry in single molecular bonds. Annu Rev Biophys Biomol Struct 2001;30:105-128

20 Evans E, Leung A, Hammer D, Simon S. Chemically distinct transition states govern rapid dissociation of single L-selectin bonds under force. Proc Natl Acad Sci U S A 2001;98(07): 3784-3789

21 Webb B, Sali A. Comparative protein structure modeling using MODELLER. Curr Protoc Bioinformatics 2016;54:1

22 Cai Z, Yarovoi SV, Zhu Z, et al. Atomic description of the immune complex involved in heparin-induced thrombocytopenia. Nat Commun 2015;6:8277

23 Bourquard T, Musnier A, Puard V, et al. MAbTope: a method for improved epitope mapping. J Immunol 2018;201(10):3096-3105

24 Sachais BS, Litvinov RI, Yarovoi SV, et al. Dynamic antibodybinding properties in the pathogenesis of HIT. Blood 2012;120 (05):1137-1142

25 Rauova L, Poncz M, McKenzie SE, et al. Ultralarge complexes of PF4 and heparin are central to the pathogenesis of heparin-induced thrombocytopenia. Blood 2005;105(01):131-138

26 Bui VC, Nguyen TH. The role of single-molecule force spectroscopy in unraveling typical and autoimmune heparin-induced thrombocytopenia. Int J Mol Sci 2018;19(04):E1054

27 Cines DB, Yarovoi SV, Zaitsev SV, et al. Polyphosphate/platelet factor 4 complexes can mediate heparin-independent platelet activation in heparin-induced thrombocytopenia. Blood Adv 2016;1(01):62-74

28 Amiral J, Pouplard C, Vissac AM, Walenga JM, Jeske W, Gruel Y. Affinity purification of heparin-dependent antibodies to platelet factor 4 developed in heparin-induced thrombocytopenia: biological characteristics and effects on platelet activation. $\mathrm{Br} \mathrm{J}$ Haematol 2000;109(02):336-341 